\title{
Engineered reciprocal chromosome translocations drive high threshold, reversible population replacement in Drosophila
}

\author{
Anna Buchman, Tobin Ivy, John Marshall, Omar S Akbari, and Bruce A Hay \\ ACS Synth. Biol., Just Accepted Manuscript • DOI: 10.1021/acssynbio.7b00451 • Publication Date (Web): 02 Apr 2018
}

Downloaded from http://pubs.acs.org on April 5, 2018

\section{Just Accepted}

"Just Accepted" manuscripts have been peer-reviewed and accepted for publication. They are posted online prior to technical editing, formatting for publication and author proofing. The American Chemical Society provides "Just Accepted" as a service to the research community to expedite the dissemination of scientific material as soon as possible after acceptance. "Just Accepted" manuscripts appear in full in PDF format accompanied by an HTML abstract. "Just Accepted" manuscripts have been fully peer reviewed, but should not be considered the official version of record. They are citable by the Digital Object Identifier (DOI®). "Just Accepted" is an optional service offered to authors. Therefore, the "Just Accepted" Web site may not include all articles that will be published in the journal. After a manuscript is technically edited and formatted, it will be removed from the "Just Accepted" Web site and published as an ASAP article. Note that technical editing may introduce minor changes to the manuscript text and/or graphics which could affect content, and all legal disclaimers and ethical guidelines that apply to the journal pertain. ACS cannot be held responsible for errors or consequences arising from the use of information contained in these "Just Accepted" manuscripts. 
Engineered reciprocal chromosome translocations drive high threshold, reversible population replacement in Drosophila

Anna B. Buchman*\#, Tobin Ivy*, John M. Marshall ${ }^{\$}$, Omar S. Akbari*\#\&, and Bruce A. Hay \&

*Division of Biology and Biological Engineering, California Institute of Technology, Pasadena, CA. ${ }^{\$}$ School of Public Health, University of California Berkeley, Berkeley CA. "Division of Biological Sciences, University of California, San Diego, San Diego, CA.

\&Authors for Correspondence: haybruce@caltech.edu and oakbari@ucsd.edu

\begin{abstract}
:
Replacement of wild insect populations with transgene-bearing individuals unable to transmit disease or survive under specific environmental conditions using gene drive provides a self-perpetuating method of disease prevention. Mechanisms that require the gene drive element and linked cargo to exceed a high threshold frequency in order for spread to occur are attractive because they offer several points of control: they bring about local, but not global population replacement; and transgenes can be eliminated by reintroducing wildtypes into the population so as to drive the frequency of transgenes below the threshold frequency required for drive. Reciprocal chromosome translocations were proposed as a tool for bringing about high threshold population replacement in 1940 and 1968. However, translocations able to achieve this goal have only been reported once, in the spider mite Tetranychus urticae, a haplo-diploid species in which there is strong selection in haploid males for fit homozygotes. We report the creation of engineered translocation-bearing strains of Drosophila melanogaster, generated through targeted chromosomal breakage and homologous recombination. These strains drive high threshold population replacement in laboratory populations. While it remains to be shown that engineered translocations can bring about population replacement in wild populations, these observations suggest that further exploration of engineered translocations as a tool for controlled population replacement is warranted.
\end{abstract}

\title{
Keywords:
}

Gene drive, selfish genetic element, vector control, mosquito, malaria, dengue, UDMEL, engineered translocations, self-propagating, unbreakable, public acceptance.

One strategy for disease prevention of insect vector-borne disease, first articulated by Curtis ${ }^{1}$, involves using gene drive to bring about replacement of 
wild, disease transmitting insect populations with individuals engineered to be refractory to disease transmission, but still subject to traditional vector control (reviewed in ${ }^{2-6}$ ). An important appeal of this strategy is that it is species-specific and potentially self-perpetuating. However, gene drive mechanisms must also function within regulatory frameworks ${ }^{7-15}$. Central to these are issues of confinement and reversibility: can the spread of transgenes to high frequency be limited to locations in which their presence is sought, and can the population be restored to the pre-transgenic state ${ }^{15}$ ?

High threshold gene drive mechanisms can potentially provide positive answers to these questions. These mechanisms require that transgenes make up a large fraction of the total insect population (important examples range from 15-70\%) before they spread to high frequency within a target area in which they are broadly introduced. Below this frequency transgenes are instead actively eliminated from the population. Once replacement has occurred in the primary target area, spread to high frequency in areas connected to this region by low levels of migration is inhibited because the transgene fails to reach the threshold frequency needed for drive. Finally, transgenes can be eliminated from the population if the release of wildtypes throughout the area in which replacement has occurred results in the frequency of transgenics being driven below the threshold required for drive.

A number of gene drive mechanisms that could in principle bring about high threshold population replacement have been proposed. Examples include a number of single locus gene drive mechanisms ${ }^{16-18}$, reciprocal chromosome translocations, inversions and compound chromosomes ${ }^{19}$, and several forms of engineered underdominance ${ }^{18,20-25}$. Here we focus on the use of engineered reciprocal chromosome translocations.

A reciprocal chromosome translocation results in the mutual exchange of DNA between two non-homologous chromosomes (reviewed in ${ }^{26}$, illustrated in Figure $1 \mathrm{~A})$. Provided that the translocation breakpoints do not alter the expression and/or function of nearby genes, translocation heterozygotes and homozygotes can in principle be phenotypically normal. For example, phenotypically normal, naturally occurring translocation-bearing individuals are found in populations of many species ${ }^{27}$, including humans ${ }^{28,29}$. However, translocation heterozygotes are usually semi-sterile, producing a high frequency of inviable offspring. This occurs because meiosis in a translocation heterozygote can generate a variety of different products. Three patterns of segregation are possible: alternate, adjacent-1 and adjacent-2 (Figure 1A). While alternate segregation leads to the production of gametes with a full genome complement, adjacent-1 and adjacent2 segregation lead to the production of aneuploid gametes, resulting in the death of progeny that inherit an unbalanced chromosome set. In many species alternate and adjacent-1 segregation occur roughly equally, with adjacent-2 segregation being rare (reviewed in ${ }^{30,31}$ ). In such species progeny genotypes and survival phenotypes resulting from crosses between translocation-bearing individuals and wildtypes are as illustrated in the Punnett square in Figure 1B. 
Progeny with unbalanced genotypes die, while balanced translocation heterozygotes, translocation homozygotes, and homozygous wildtypes survive.

The frequency of a reciprocal translocation lacks a stable internal equilibrium, with either wildtype or translocation-bearing chromosomes spreading to fixation in an isolated population through natural selection. Based on this, Serebrovskii and Curtis proposed that releases of translocation-bearing individuals could be used to alter the chromosomal composition of a population ${ }^{1,32}$. Curtis went on to note that if a gene beneficial to humans could be linked to the translocation breakpoint, this behavior of translocations could be used to spread the gene to high frequency ${ }^{1}$. More recent modeling work has highlighted the potential of translocations for bringing about local, but not global population replacement, and the possibility of reversal to the pre-transgenic state ${ }^{33}$. The positive points notwithstanding, it is important to note that wide-scale spread is only expected under a limited set of conditions. Thus, modeling suggests that in spatially distributed populations underdominant alleles must convey a fitness benefit in order to spread from a localized introduction, as traveling waves ${ }^{34,35}$. These authors also note that the spatial dynamics of bistable systems depend critically when considering spread from a point source - on factors such as local differences in population density and migration rate. Since it is unlikely that underdominant systems such as reciprocal translocations will confer a fitness benefit to carriers, these observations imply that population replacement strategies involving translocations will need to utilize an alternative approach, in which translocations are distributed more or less uniformly throughout the target area at super threshold levels.

Evolutionary studies show that translocations can become fixed in populations ${ }^{27}$. However, efforts to directly bring about population replacement using translocations created in the lab have generally not been successful (reviewed in $19,30,36,37$ ). Thus, for example, Robinson and Curtis found that even 9:1 introduction ratios of fit-seeming translocation homozygotes into wildtype Drosophila populations resulted in elimination of the translocation from the population ${ }^{36}$. In most other experiments, in a variety of insects, homozygotes were unfit, rare or entirely absent, indicating low fitness ${ }^{30,37-39}$. This low fitness could sometimes be ameliorated through extensive introgression into wildtype strains ${ }^{40}$, though the introgressed translocations were never tested for ability to bring about population replacement. Field tests of population replacement using Aedes aegypti homozygous for a translocation were unsuccessful ${ }^{41}$. The CSIRO Entomology group did achieve some success in population reduction with small field trials of translocation-bearing Australian Blow flies, but later larger-scale trials failed and the efforts were ultimately abandoned (reviewed in ${ }^{42,43}$ ). Another group, working with the spider mite Tetranychus urticae, has shown population replacement with a small fraction of translocations generated. However, this species likely represents something of an exception since its haplodiploid lifecycle (in which males develop from unfertilized eggs) provides a strong selective filter for translocations that are likely to be viable and fit as homozygotes ${ }^{44}$. Several reasons are likely to account for why most 
translocations tested fail to drive. First, the translocation-bearing individuals (particularly homozygotes) were generated using X-rays. This can result in a high frequency of background mutations, which can reduce fitness, particularly of homozygotes (reviewed in ${ }^{30,37}$ ). Second, breakpoints may disrupt genes or their regulatory regions. Finally, more recently it has become clear that chromosome positioning and structure in the nucleus can play a role in determining large-scale patterns of gene expression, and that chromosome translocation can result in changes in the patterns of gene expression ${ }^{45,46}$. These changes also may result in translocation-bearing individuals experiencing a fitness cost. These latter observations in particular leave it unclear how frequent translocation-bearing individuals of high fitness are. To explore these issues we developed an approach to generate and identify site-specific reciprocal chromosomal translocations. We report the generation of two strains of Drosophila carrying engineered chromosome translocations and show they are capable of bringing about threshold-dependent population replacement in competition with a laboratory wildtype strain. Implications of these results and next steps are discussed.

\section{Results \\ Engineering Reciprocal Translocations in Drosophila}

Cells or organisms carrying translocations with defined breakpoints have recently been generated using several strategies. One set of approaches begins with two non-homologous chromosomes that each have a different transgene-bearing cassette inserted at a specific position. Recombination between the two chromosomes to generate a translocation is then driven by FLP/FRT recombination ${ }^{47}$, Cre/loxP recombination ${ }^{48,49}$, or homologous recombination following double-stranded break creation within the transgene cassettes using a site-specific nuclease ${ }^{49-51}$. Translocations have also been generated in completely wildtype backgrounds, following CRISPR/Cas9-mediated cleavage of two otherwise wildtype chromosomes followed by non-homologous end joining 52-54. In this latter case, PCR-based methods were used to identify pools of cells or individual $C$. elegans carrying translocations.

We sought to create translocations using a variant of the approach described by Egli et al. in which homologous recombination between two chromosomes follows double-stranded break creation using the rare-cutting site-specific nuclease I-Scel ${ }^{49}$. However, rather than use their approach for identification of potential translocation bearing individuals, which involves scoring for the loss of the marker $y+$ in an otherwise a $y$ - background, we created a system in which recombination results in the creation of a dominant marker. This approach can be used in otherwise wildtype genetic backgrounds, in diverse species.

Two constructs ( $\mathrm{A}$ and $\mathrm{B}$ ) were generated (Figure 2B). Each construct included several components. These were (from left to right) a transformation marker (the white gene); a location that could be used as an insertion point of a gene of interest (GOI); a promoter that drives the expression of a dominant fluorescent 
marker, either ubiquitously (the Opie2 viral promoter ${ }^{55}$ ) or in oenocytes ${ }^{56}$; a splice donor site, and two stretches of DNA used as substrates for homologous recombination, annotated as UVW and XYZ, each roughly 670bp in length. These DNA fragments were derived from the mouse IgG locus, and thus lack homology with the Drosophila genome. Two target sites for the rare cutting homing endonuclease I-Scel were inserted between UVW and XYZ. To the right of these elements were positioned a splice acceptor, a promoterless reporter gene (GFP or dsRed), and a phiC31 recombination attB site.

These constructs were introduced into flies at three separate attP locations: construct $A$ at $51 \mathrm{C}$ on chromosome 2, and construct $B$ at $68 \mathrm{E}$ or $70 \mathrm{~A} 2$ on chromosome 3 (Figure 2A). The attP insertion sites at 51C and 68E lie some distance from annotated genes, while the 70A2 site lies within a cluster of tRNA loci. Both constructs were oriented in the same direction with respect to their centromeres (Figure 2A). The constructs were designed so that flies bearing construct $A$, located on the second chromosome, would express the svp-driven eGFP marker, while construct $B$, located on the third chromosome, would express the opie2-driven dsRED marker (Figure 2B). Transgenics for construct B behaved as expected, and were dsRED positive throughout their body. However, transgenics for construct $A$ had no detectable GFP expression. The basis for this is unclear, but could be due to inappropriate splicing of the XYZ-UVW sequence in this construct. Regardless, as illustrated below, one marker is sufficient to identify translocation-bearing individuals.

To generate translocation-bearing individuals we generated stocks doubly homozygous for constructs $A$ and $B(51 \mathrm{C} ; 71 \mathrm{~A} 2$ or $51 \mathrm{C} ; 68 \mathrm{E})$. These were then mated with flies that express I-Scel under the control of the Hsp70 heat shock promoter ${ }^{57}$. Progeny harboring all three transgenes were subjected to multiple rounds of heat shock during larval stages and as adults. Adults were outcrossed to wildtype, and progeny examined under a fluorescent dissecting scope. In a number of individuals strong ubiquitous GFP expression was observed. This is the predicted outcome if I-Scel expression results in cleavage of both transgenebearing chromosomes (Fig. 2C), followed by homologous recombination between $\mathrm{XYZ}$ - and UVW-bearing ends of the two different chromosomes (Fig. 2D,E). Putative translocation heterozygotes $\left(T_{1} /+; T_{2} /+\right)$ were individually mated to wild type individuals $(+/+;+/+)$ to generate males and female translocation heterozygotes (identified as GFP-expressing). These were mated with each other to generate putative translocation homozygotes $\left(T_{1} / T_{1} ; T_{2} / T_{2}\right)$. PCR and sequencing of products from genomic DNA of these individuals was used to demonstrate that these individuals were homozygous for both translocation products (Methods and Figure 2F).

To explore the genetic behavior of translocation-bearing chromosomes and the fitness of carriers we performed a number of crosses and quantified progeny genotype (Table 1). Stocks consisting of translocation homozygotes appeared generally healthy as adults, and survival from egg to adult was $96 \%$ of that 
observed for the Canton S (CS) wildtype stock. In contrast, crosses between males or females heterozygous for the translocation and wildtype resulted in semisterility, with only about $50 \%$ of progeny surviving to adulthood, and $50 \%$ of the survivors being translocation heterozygotes. These are the expected results if alternate and adjacent-1 segregation occur with equal frequency in translocationbearing individuals during meiosis, resulting in the production of $50 \%$ aneuploid gametes (Figure 1B). Finally, for each translocation type we also carried out crosses between male and female translocation heterozygotes. Only $37.5 \%$ of progeny are predicted to survive, due to the large fraction of zygotes carrying unbalanced chromosome complements. However, many of the survivors $(83 \%)$ are predicted to carry one or two copies of the translocation (Figure 1B). The levels of embryo survival and percentage of adults carrying the translocation were in good agreement with these predictions (Table 1). Together, these observations suggest that the translocation-bearing strains are fit (notwithstanding the expected semisterility), at least to a first approximation. These points notwithstanding, fitness measurements such as these are not sufficient to know that frequency-dependent drive will occur. This is well illustrated by the results of Curtis and Robinson, who found that a 2;3 translocation strain generated with X-rays, which had homozygous viability and fertility equivalent to wildtype in crosses such as those described above, was unable to drive population replacement, even when introduced at a 9:1 translocation:wildtype ratio ${ }^{36}$.

For population replacement experiments we first introgressed our translocationbearing systems, 51C; 70A2 and 51C; 68E flies, with Canton S (CS) for 8 generations, so as to minimize background genetic differences between translocation-bearing and wildtype strains. Translocation-bearing individuals were then backcrossed to each other to create homozygous stocks, which were then expanded and maintained for use in population experiments. We initiated population cage experiments by introducing translocation-bearing males and virgin females into cages along with Canton $\mathrm{S}$ males and virgin females of similar age. A number of different introduction frequencies were tested, in triplicate. These included frequencies predicted to be super-threshold $(80 \%, 70 \%, 60 \%)$, and sub-threshold $(20 \%, 30 \%, 40 \%)$. Populations were then followed for 14 generations, with the frequency of translocation-bearing individuals noted each generation.

Results of these experiments are summarized in Figure 3A,B (solid lines). For both translocation-bearing strains, all nine releases at frequencies lower than $50 \%$ resulted in elimination of the translocation from the population. Conversely, introductions at frequencies greater than $50 \%$ resulted in translocation-bearing genotypes spreading to high frequency. These results are generally consistent with modeling predictions. However, the dynamics of drive are clearly distinct from those predicted for translocations that lack a fitness cost (dotted lines in Figure $3 A, B$ ). When translocations were introduced at predicted super-threshold frequencies spread was slower than expected for a translocation with no fitness 
cost. Sub-threshold releases also resulted in lower initial translocation frequencies than expected, and this was generally followed in later generations by a modestly decreased time to elimination as compared with a translocation with no fitness cost, except at the $20 \%$ introduction frequency.

We explored a number of alternative fitness cost models in order to provide better agreement between the laboratory drive data and theoretical model predictions (Supplementary Text S1). The model that provided the best fit to the observed data was one in which lab-reared individuals homozygous for the translocation and their translocation homozygote offspring had reduced fitness if they were not the result of outbreeding with wild-type individuals (Figure S1). However, significant discrepancies between the observed and predicted dynamics remained, suggesting that further experimental and modeling work will be required to understand these discrepancies and any mechanisms that may be responsible for them.

\section{Predicted tradeoffs associated with translocation-based gene drive.}

In real world scenarios other than initial field-testing - in which population isolation will be essential - there will be some level of reciprocal migration between the target area for population replacement and surrounding areas. An important feature of translocations, as distinct from some other proposed forms of underdominance-based gene drive ${ }^{16-18,20-22}$, is that heterozygotes are viable and fertile, which creates opportunities for the flow of transgenes into neighboring wildtype populations, and wildtype alleles into the replaced population. This behavior has been briefly considered by Marshall and Hay ${ }^{33}$. Here we use this framework to consider in more detail the scenario in which the target population for replacement (population 1) and a second, similarly sized population (population 2) are linked by equal levels of reciprocal migration. Previous modeling studies of underdominant systems have noted that the presence of reciprocal migration can result in internal equilibria containing both wildtype and underdominant alleles $21-23,33,58$. Other studies have explored the fate of underdominant alleles in interacting populations in which alleles are first introduced into a local area and then spread outward ${ }^{34,35}$. Here we consider the case of reciprocal translocations specifically, in which translocation introductions have initially been carried out throughout population 1 .

Figure 3A illustrates a specific scenario, in which translocation homozygotes with no fitness cost are introduced into population 1 at a frequency of $70 \%$ for three consecutive generations, and are connected to a similarly sized population 2 by a migration rate of $1 \%$. The translocation increases to high frequency $(\sim 99 \%)$ in population 1, but not to allele (all are translocation homozygotes) or genotype (all are translocation heterozygotes or homozygotes) fixation, since wildtypes are introduced into population 1 each generation from population 2. Translocationbearing genotypes are also present at modest levels $(<5 \%(4.99 \%))$ in population 2. Figure $3 \mathrm{~A}$ also illustrates an identical scenario in which the migration rate is now $5 \%$. In this case the translocation equilibrium frequency is $<95 \%(94.67 \%)$ in 
population $1,<25 \%(24.49 \%)$ in population 2 . We ran this model across a range of fitness costs and migration rates to see the general relationship between fitness cost, migration rate and equilibrium frequency in populations 1 and 2 (Figures $3 \mathrm{~B}$ and $\mathrm{C}$, same release conditions as for the single run shown in Figure $3 A)$. The highest level of incoming wildtype migration that can be tolerated for a translocation with no fitness cost $(\sim 7.0 \%$ / generation) results in an equilibrium translocation genotype frequency of $\sim 90 \%$ in population 1 and $\sim 25 \%$ in population 2. Decreased levels of migration result in correspondingly higher equilibrium frequencies within population 1 , which approach fixation as the migration rate falls to zero (Figure 4A), and the converse holds true for population 2. Increased fitness costs result in a minimal decrease in equilibrium translocation frequency for both populations compared to changes in migration rate, as seen by the sharper change in shading along the $\mathrm{Y}$-axis (migration rate) than along the X-axis (fitness cost) (Figure 4B,C).

Population size is also a consideration as an equivalent stochastic model implemented by Marshall and Hay ${ }^{33}$ for a translocation with a homozygous fitness cost of $5 \%$ and heterozygous fitness cost of $2.5 \%$ showed, for two populations of 100 individuals, the system had a $\sim 5 \%$ chance of becoming established in both populations for a migration rate of $6.0 \%$ per generation; however for two populations of 1,000 individuals, there was only a small chance $(\sim 0.1 \%)$ that the system became established in both populations for the same migration rate. These observations suggest there is a broad range of conditions under which translocations can spread to a local high frequency, but highlight the tradeoffs associated with increased levels of migration between target and neighboring populations.

\section{Discussion}

Translocations have previously been generated in animals and plants in several ways using transgenesis, though the fitness of individuals carrying these chromosomes has not been characterized ${ }^{47-51}$. The tools we used to create translocations in Drosophila - transgene cassettes located on two different chromosomes, a dominant marker created through the act of translocation, a site-specific nuclease able to bring about breakage within each cassette, and unique sequences that can mediate recombination between the two chromosomes - should be portable to other species (at least ones where sufficient mapping data and genome sequence are available). In particular, future use of the Cas9 system will allow the creation of double-stranded breaks at userdefined sites, which should facilitate the generation of translocations with breakpoints chosen by the developer ${ }^{5}$. The crossing scheme required to generate translocations can also be simplified to a single cross through the use of pairs of chromosomes, one of which carries Cas9, and the other of which carries a gRNA, the combination of which results in site-specific nuclease activity that cleaves a target site present on both transgene-bearing chromosomes $(A B$, $\mathrm{OSA}$, and $\mathrm{BAH}$, unpublished). These features, coupled with the common genetic behavior of reciprocal translocations in diverse species (semisterility in 
heterozygotes), suggest that translocation-based, high threshold and reversible drive may be possible in many species.

Translocations generated in the past, with one exception in a haploid-diploid species ${ }^{44}$, have not been shown to drive population replacement. This is likely due, at least in part, to the creation of background mutations that compromise fitness in response to the X-irradiation used to create them. Our observations demonstrating population replacement at high but not low introduction frequencies, while limited to two translocations sharing one breakpoint in common, suggest that it may be possible to generate engineered translocations with fitness comparable to wildtype laboratory strains. That said, while the translocations we generated are competitive in a constant laboratory environment, it remains to be shown that these or any other engineered translocations are fit in competition with the diversity of genotypes that would be encountered in complex natural environments.

The population dynamics associated with the spread or loss of our translocations highlight this last point. Both translocations share a common breakpoint and show similar population dynamics. Thus, these dynamics may reflect breakpointspecific effects on gene expression. Alternatively, and/or in addition, they may reflect the continued segregation of fitness modifiers during drive, since recombination on translocation-bearing chromosomes in Drosophila is reduced throughout the involved arms ${ }^{59}$. Understanding the basis for these dynamics, and whether they are specific to these translocation breakpoints and/or the dominant markers used, will require further study in other genetic backgrounds, and with other engineered translocations, work that is in progress.

Our modeling also illustrates a set of tradeoffs associated with translocationbased gene drive. While an increase in translocation to high frequency can be spatially limited to a single population, this comes with the cost that wildtypes are continuously being introduced into the replaced population, and transgenes are introduced into the neighboring population. This flow keeps the equilibrium frequency of transgene-bearing individuals below $100 \%$ in the replaced population and above zero in the neighboring population. These observations suggest that translocation-based gene drive is likely to be most epidemiologically effective, and able to satisfy regulatory requirements relating to the presence and movement of transgene-bearing organisms, in target areas circumscribed by significant barriers to migration.

Related to these points, an important insight gained from other modeling of underdominant systems in spatially distributed populations is that if area-wide population replacement is attempted, attention must be paid to the population dynamics at hybrid zones near borders, as the hybrid front (a traveling wave) can move over time, and depends importantly on the distribution of population densities and migration distances inward and outward ${ }^{34,35}$. Thus what constitutes a border may often literally be a moving target. Maintenance of specific borders 
will require monitoring and potentially entail additional local releases of the translocation system or wildtype inside or outside the target area, respectively. Modeling of translocation behavior using spatially explicit models based on analysis of real populations in complex environments should provide further insight into the likely behavior of these entities in real populations ${ }^{60,61}$. Finally, mosquito populations in the wild sometimes consist of multiple chromosomal forms, and may also display some level of reproductive isolation ${ }^{62-64}$. How engineered translocations will fare in the face of these variants remains to be determined, but can be explored in competition with genetically diverse laboratory strains ${ }^{65,66}$. While an understanding of the above issues is critical for the success of any population-replacement strategy, the problems may not be intractable, as evidenced by successes in controlling pest populations using nontransgenic ${ }^{67}$ and transgenic inundative population suppression strategies ${ }^{68,69}$.

Finally, we address possible sources of failure and ways in which translocationbased drive can potentially overcome them. Pathogens can evolve resistance to the activities conferred by the cargo transgene, and the transgene can mutate to inactivity. These events cannot be prevented, but chromosome-based drive mechanisms such as translocations have the attractive feature that it should be possible to incorporate multiple transgenes near the breakpoints, bringing about redundancy in effector function and thereby increased functional lifetime in the wild. Cycles of population replacement to bring new genes into the population can also be imagined. In one approach, the translocation can first be removed from the population by driving its frequency below the threshold needed for drive throughout the target area, through dilution with wildtypes. This can then be followed by a second release of a new translocation-bearing strain that has the same breakpoints, and a new cargo. Alternatively, if high fitness translocations with distinct breakpoints can be generated routinely, it may be possible to drive a first generation translocation and any remaining wildtypes out of the population in favor of a second, distinct translocation (a point made earlier by ${ }^{32}$ ) in the context of use of translocations for population suppression) carrying a new cargo, as with proposals for cycles of replacement of Medea-based gene drive systems ${ }^{3,70-72}$. The translocation itself is likely to be evolutionarily stable as a drive vehicle since reversion back to the wildtype chromosome configuration is likely to be very rare. However, even if this happened, necessarily in a single rare individual, this chromosome would be eliminated along with other wildtype chromosomes in a population (of this or any other species (see above)) in which the translocation was present at high frequency.

\section{Materials \& Methods: Construct Assembly}

The Gibson enzymatic assembly (EA) cloning method was used for all cloning ${ }^{73}$. For both constructs (A and $B$ ), translocation allele components were cloned into the multiple cloning site (MCS) of a plasmid ${ }^{74}$ containing the white gene as a marker and an attB-docking site. For construct A (Figure 1B), the oenocytespecific svp enhancer ${ }^{56}$ and Hsp70 basal promoter fragments were amplified 
from Drosophila melanogaster genomic DNA using primers P16 and P17 (svp) and P18 and P19 (Hsp70). The GFP fragment was amplified from template pAAV-GFP (addgene plasmid \#32395) using primers P26 and P27. A Kozak sequence (CAACAAA) directly 5' of the GFP start codon was added with primer P26. The SV40 3'UTR fragment was amplified from template pMos-3xP3-DsRedattp (addgene plasmid \#52904) using primers P28 and P10. The 5' and 3' CTCF insulator fragments ${ }^{75}$ were amplified from Drosophila melanogaster genomic DNA using primers P11 and P15 (for the 5' CTCF fragment) and P13 and P14 (for the 3' CTCF fragment). The $667 \mathrm{XYZ}$ and 668 UVW homology fragments were amplified as above with primers P22 and P23 (XYZ) and P20 and P21 (UVW), from plasmid pFUSE-mlgG1-Fc Invivogen, San Diego). The 5' and 3' splice sites utilized were from a $67 \mathrm{bp}$ intron located in the Drosophila melanogaster Myosin Heavy Chain (MHC) gene ID CG17927. They were added to UVW and XYZ sequences using PCR; the 5' splice site was added to the 5' end of the UVW fragment via PCR with primer P24, and the 3' splice site was added to the 3' end of fragment XYZ via PCR with primer P25. Two I-Scel recognition sequences Two 18bp I-Scel recognition sequences (ATTACCCTGTTATCCCTA-CTAG-TAGGGATAACAGGGTAAT) were added to the 3' end of the UVW fragment with primer P21 and the 5' end of the XYZ fragment with primer P22. The construct was assembled in two steps, as above, with the first (5') CTCF, the svp and hsp70 fragments, the UVW fragment, and the $X Y Z$ fragment cloned in via a first EA cloning step, and the GFP fragment, the SV40 3'UTR fragment, and the second (3') CTCF cloned in via a second EA cloning step. For construct $B$ (Figure 1B), the opie2 promoter fragment was amplified from plasmid plZ/V5-His/CAT (Invitrogen) using primers P1 and P2. The XYZ and UVW homology fragments were amplified from plasmid pFUSEssCHIg-mG1 using primers $\mathrm{P} 3$ and P4 (XYZ) and P5 and P6 (UVW). Two 18bp IScel recognition sequences (ATTACCCTGTTATCCCTA-CTAGTAGGGATAACAGGGTAAT) were added to the 3' end of the XYZ fragment and the 5' end of the UVW fragment in inverse orientation to each other separated by a $4 \mathrm{bp}$ linker sequence (CTAG) using primers P4 (for XYZ) and P5 (for UVW). The 5' and ' '' splice sites utilized were from a $67 \mathrm{bp}$ intron located in the Drosophila melanogaster Myosin Heavy Chain (Mhc) gene ID CG17927; the 5' splice site was added to the 5' end of the XYZ fragment via PCR with primer P7, and the 3' splice site was added to the 3' end of fragment UVW via PCR with primer P8. The dsRed fragment, together with the SV40 3'UTR, were amplified from template pMos-3xP3-DsRed-attp (addgene plasmid \#52904) using primers P9 and P10, with a Kozak sequence (CAACAAA) directly 5' of the DsRed start codon added with primer P9. The 5' and $3^{\prime}$ CTCF insulator fragments ${ }^{75}$ were amplified from Drosophila melanogaster genomic DNA using primers P11 and P12 (for the 5' CTCF fragment) and P13 and P14 (for the 3' CTCF fragment). The construct was assembled in two steps. First, the Drosophila melanogaster attB stock plasmid ${ }^{74}$ was digested with Ascl and Xbal, and the first (5') CTCF, the opie-2 promoter, the XYZ fragment, and the UVW fragments were cloned via EA cloning. Then, the resulting plasmid was digested with Xhol, and the dsRedSV40 3'UTR fragment and the second (3') CTCF were cloned in via EA cloning. 
All sequences were analyzed with NNSPLICE 0.9 (available at http://www.fruitfly.org/seq_tools/splice.html) to confirm strength of splice signals and to check for cryptic splice sites. A list of primer sequences used in the above construct assembly can be found in Supplementary Table 1.

\section{Fly Culture and Strains}

Fly husbandry and crosses were performed under standard conditions at $25^{\circ} \mathrm{C}$. Rainbow Transgenics (Camarillo, CA) carried out all of the fly injections. Bloomington Stock Center (BSC) fly strains utilized to generate translocations were attP lines 68E (BSC \#24485: $y^{1}$ M\{vas-int.Dm\}ZH-2A w*; M\{3xP3RFP.attP'\}ZH-68E), 51C (BSC \#24482; y[1] M\{vas-int.Dm\}ZH-2A w[]; M\{3xP3RFP.attP'\}ZH-51C), and 70A2 (BSC \#9741: y[1] w[1118]; PBac\{y[+]-attP9A\}VK00023). Fly Stock BSC\#6935 (y[1] $\left.\quad w{ }^{*}\right] ; \quad P\{r y[+t 7.2]=70 F L P\} 23$ $\mathrm{P}\{\mathrm{v}[+\mathrm{t} 1.8]=70 \mathrm{I}-\mathrm{Sce}\} \mathrm{AA} / \mathrm{TM})$ was used as the source of heat shock induced IScel. For balancing chromosomes, fly stocks BSC\#39631 (w[*]; wg[Sp-1]/CyO; $\mathrm{P}\{\mathrm{ry}[+\mathrm{t} 7.2]=$ neoFRT\}82B Isn[SS6]/TM6C, Sb[1]) BSC\#2555 (CyO/sna[Sco]) were used. For introgression into a wild type background we used the Canton-S stock BSC\#1. Translocation construct A was inserted at site $51 \mathrm{C}$, and construct $B$ was inserted at 68E and 70A2 using phiC31 mediated attP/attB integration. These site combinations allowed for the generation of two distinct translocation types, 51C;68E and 51C;70A2. Stocks homozygous for both constructs were then mated with flies that express I-Scel under the control of the Hsp70 heat shock promoter ${ }^{57}$. Progeny carrying all three transgenes were subjected to 5 rounds of heat shock during larval stages and as adults. Heat shocks were conducted by submerging fly vials in a water bath set to $38^{\circ} \mathrm{C}$ for one hour. Adults were outcrossed to w-, and progeny examined under a fluorescent dissecting scope for ubiquitous GFP expression, indicative of translocation generation.

Homozygous translocation-bearing stocks were generated for both $51 \mathrm{C} ; 68 \mathrm{E}$ and 51C;70A2 site combinations by crossing translocation heterozygotes and identifying homozygous progeny by eye color (light orange eyes for homozygotes versus yellow for heterozygotes for the 51C;68E site combination; light red eyes for homozygotes versus orange for heterozygotes for the 51C;70A2 site combination. After confirming homozygous viability, translocations were introgressed into a Canton-S genetic background. First, CS females were crossed to translocation-bearing males so as to bring the CS mitochondrial genotype into the translocation background. Subsequently, translocation heterozygote females were outcrossed to CS males for 8 generations. Heterozygous translocation-bearing males and virgin females were then crossed to each other to generate homozygous stocks in the CS background for each site combination. Homozygosity was confirmed by outcrossing. Drive experiments for these stocks were set up against CS as the wildtype stock.

\section{Embryo and Adult viability determination}

For embryo viability counts (Table 1), 2-4 day old adult virgin females were mated with males of the relevant genotypes for 2-3 days in egg collection 
chambers, supplemented with yeast paste. On the following day, a $3 \mathrm{hr}$ egg collection was carried out, after first having cleared old eggs from the females through a pre-collection period on a separate plate for 3hrs. Embryos were isolated into groups and kept on an agar surface at $25^{\circ} \mathrm{C}$ for $48-72 \mathrm{hrs}$. The $\%$ survival was then determined by counting the number of unhatched embryos. One group of 100-300 embryos per cross was scored in each experiment, and each experiment was carried out in biological triplicate (total number of offspring scored is presented in Table 1). The results presented are averages from these three experiments. Embryo survival was normalized with respect to the \% survival observed in parallel experiments carried out with the Canton-S wild-type strain, which was $93.00 \%+1.82 \%$. For adult fly counts (Table 1 ), individual flies for each genotype cross were singly mated. For each genotype cross, we set up 10-15 individual fly crosses, and the results presented are averages from all these experiments (total number of offspring scored is presented in Table 1). $\chi^{2}$ statistical analyses were carried out for both embryo and adult fly counts to compare expected and observed values, and no statistically significant differences were observed ( $p$ values shown).

\section{Population cage experiments}

All population cage experiments were carried out at $25^{\circ} \mathrm{C}, 12$ hour- 12 hour day night cycle, with ambient humidity in $250 \mathrm{ml}$ bottles containing Lewis medium supplemented with live, dry yeast. Starting populations for drive experiments included equal numbers of virgins and males of similar ages, for each genotype. Translocation-bearing homozygotes were introduced at population frequencies of $60 \%, 70 \%$, and $80 \%\left(\mathrm{~T}_{1} / \mathrm{T}_{1} ; \mathrm{T}_{2} / \mathrm{T}_{2}\right)$ for above threshold drive experiments, and $20 \%, 30 \%$, and $40 \%\left(\mathrm{~T}_{1} / \mathrm{T}_{1} ; \mathrm{T}_{2} / \mathrm{T}_{2}\right)$ for below threshold drive experiments. CS virgin females and males $(+/+;+/+)$ of similar age as the translocation-bearing individuals made up the remainder of the population. The total number of flies for each starting population was 100 . All experiments were conducted in triplicate. After being placed together, adult flies were removed after seven days. After another seven days, progeny (typically 200-250, depending on the replicate) were collected and divided arbitrarily into two equally sized groups. For one group the fraction of translocation-bearing individuals $\left(T_{1} / T_{1} ; T_{2} / T_{2}\right.$ or $\mathrm{T}_{1} /+; \mathrm{T}_{2} /+$ ) was determined, while the other group was placed into a new bottle to initiate the next generation. No significant evidence of crowding in the $250 \mathrm{ml}$ bottles was observed.

\section{Theoretical Framework}

We apply the model of Curtis and Robinson ${ }^{76}$ to describe the spread of reciprocal translocations through a population. This is a discrete-generation, deterministic population frequency model assuming random mating and an infinite population size. We denote the first chromosome with a translocated segment by " $T$ " and the wild-type version of this chromosome by " $t$." Similarly, we denote the second chromosome with a translocated segment by " $R$ " and the wildtype version of this chromosome by "r." As a two-locus system, there are nine possible genotypes; however, only individuals carrying the full chromosome 
complement are viable, which corresponds to the genotypes TTRR, TtRr and ttrr, the proportion of the $k$ th generation of which are denoted by $p_{k}^{T T R R}, p_{k}^{T R R r}$ and $p_{k}^{t t r r}$. The four haplotypes that determine the genotype frequencies in the next generation $-T R, t R, T r$ and $t r$ - are described by the following frequencies:

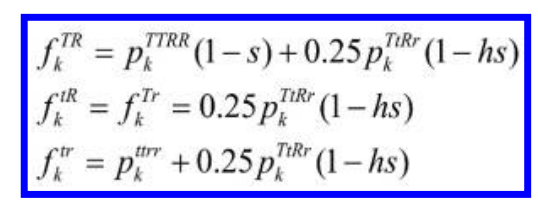

Here, $s$ denotes the reduced fecundity of TTRR individuals and $h s$ denotes the reduced fecundity of $T t R r$ individuals relative to wild-type individuals, where $h \in[0,1]$. By considering all possible mating pairs, the genotype frequencies in the next generation are:

$$
\begin{aligned}
& p_{k+1}^{T T R}=\left(f_{k}^{T R}\right)^{2} / \sigma_{k} \\
& p_{k+1}^{T R r}=2\left(f_{k}^{T R} f_{k}^{t r}+f_{k}^{t R} f_{k}^{T r}\right) / \sigma_{k} \\
& p_{k+1}^{t r r}=\left(f_{k}^{t r}\right)^{2} / \sigma_{k}
\end{aligned}
$$

where $\sigma_{k}$ is a normalizing term given by,

$$
\sigma_{k}=\left(f_{k}^{T R}\right)^{2}+2\left(f_{k}^{T R} f_{k}^{t r}+f_{k}^{i R} f_{k}^{T r}\right)+\left(f_{k}^{i r}\right)^{2}
$$

We evaluated a number of fitness cost models in terms of their ability to replicate the dynamics observed in the laboratory drive experiments. These included: a) constant fitness costs, b) fitness costs that varied with translocation population frequency, c) fitness costs that decreased with time (either linearly, exponentially or sigmoidally), and d) an introgression model, in which lab-reared individuals homozygous for the translocation and their translocation homozygote offspring had reduced fitness if they were not the result of outbreeding with wild-type individuals. These models are described in Supplementary Text S1.

For our three-population models, there are three sets of the above equations to represent each population. We let $m$ represent the migration rate per generation. After genotype frequencies for all three populations are calculated for a given generation, a proportion $m$ is removed from each genotype from populations 1 and 3 and added to population 2, and a proportion $2 m$ is removed from each genotype from population 2, half of which is added to population 1 and the other half of which is added to population 3.

\section{Funding Sources}

Work in BAH's lab (BAH, OSA, ABB, and TI) was supported by the U. S. Army Research Laboratory and the U. S. Army Research Office under contract W911NF-11-2-0055 to the California Institute of Technology, and the USDA and CRDF. Work at UCSD (OSA and AB) was supported by an NIH-K22 Career Transition award (5K22Al113060), an NIH Exploratory/Developmental Research 
Grant Award (1R21Al123937), and a Defense Advanced Research Project Agency (DARPA) Safe Genes Program Grant (HR0011-17-2-0047) awarded to O.S.A. JMM was supported by funding from The Parker Foundation through a gift to the University of California, San Francisco, Global Health Group Malaria Elimination Initiative. TI was supported by NIH training grant 1432 .

Conflicts of Interest

A.B, O.S.A and B.A.H have a patent pending related to population control using engineered translocations.

\section{Author Contribution(s)}

A.B., O.S.A, B.A.H conceived and designed experiments. T.I, J.M.M, A.B., O.S.A performed all mathematical, molecular and genetic experiments. All authors analyzed the data, contributed to the writing, and approved final manuscript.

\section{Supporting Information}

Includes Supplementary Methods describing the general theoretical framework of the modelling used and the specifics and comparison of various investigated fitness cost models, as well as Supplementary Figure 1 and Supplementary Table 1. 


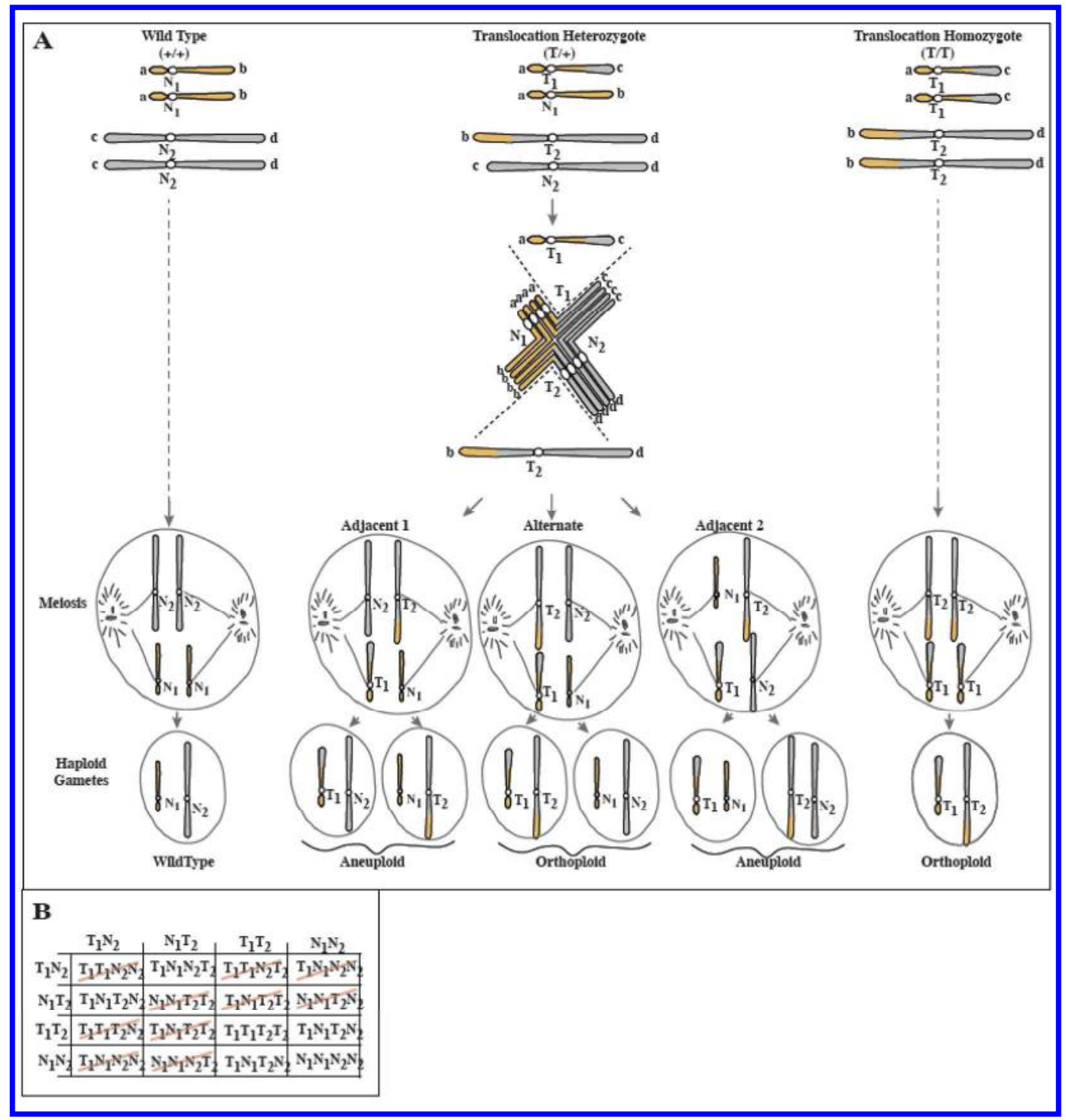

Figure 1. Gamete and zygote genotypes associated with the presence of a reciprocal translocation. Wildtype chromosomes $\mathrm{N}_{1}$ and $\mathrm{N}_{2}$, and translocation chromosomes $T_{1}$ and $T_{2}$, are indicated. (A) One chromosome type (a) is indicated in yellow. A second chromosome type (b) is in gray. Gamete types generated by wildtype $(+/+)$, translocation heterozygotes $(\mathrm{T} /+)$, and translocation homozygotes (T/T) are indicated. (B) Gamete and zygote genotypes possible in crosses involving a translocation are indicated. Inviable genotypes are indicated by a red line. 


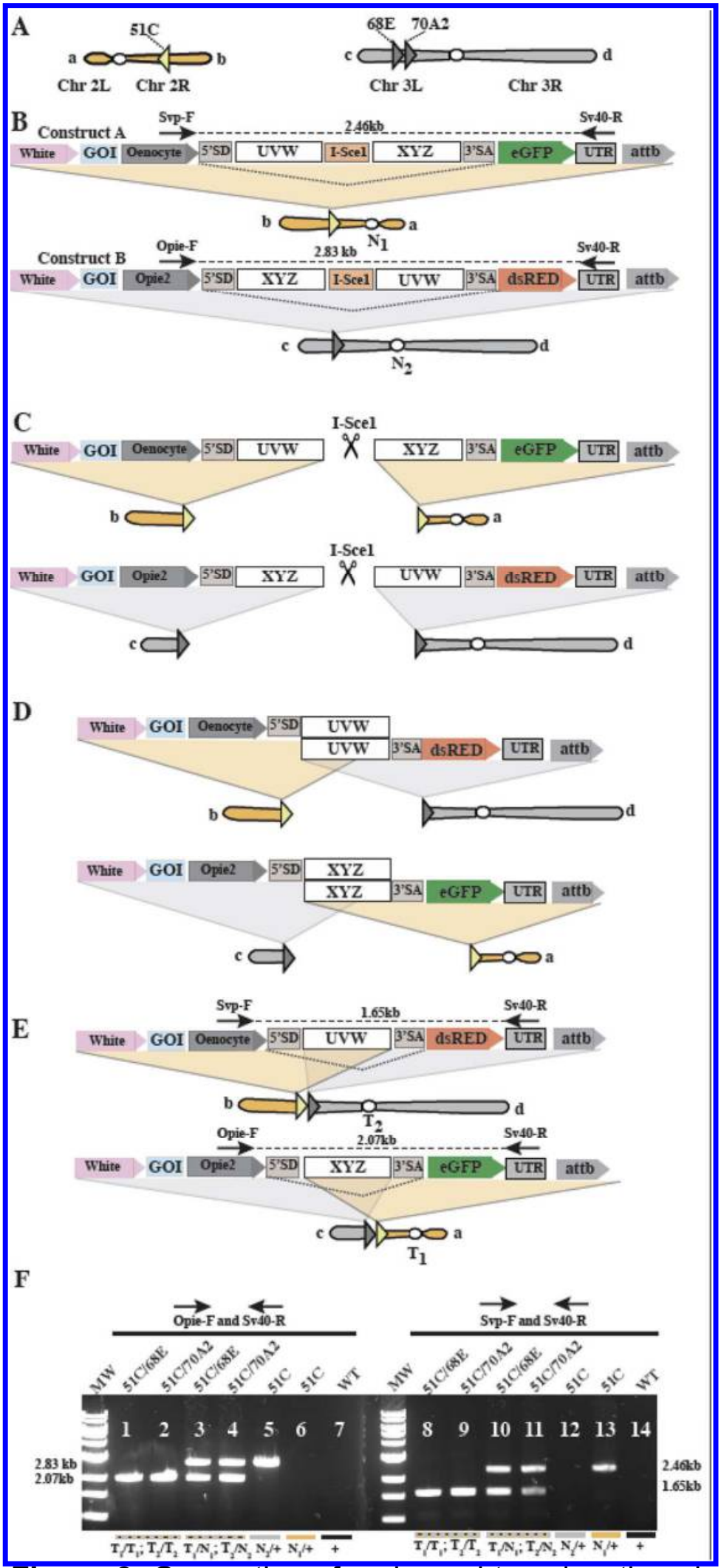

Figure 2. Generation of reciprocal translocations in Drosophila. (A) Approximate location of the attP sites used for transgene insertion; orientation with respect to 
the centromere are indicated by triangles. (B) Components of each starting transgene cassette. Construct $A$ is inserted on the second chromosome and construct $\mathrm{B}$ on the third chromosome. Components are as indicated in the text. (C) I-Sce-dependent cleavage results in a double-stranded break in each transgene-bearing chromosome. (D) Alignment of broken chromosome ends occurs using homologous sequences UVW and XYZ. (E) Recombinant chromosomes are generated by homologous recombination using sequences UVW and XYZ. (F) Agarose gel image is shown of PCR amplification products generated from different genotypes: translocation homozygotes (T1/T1; T2T2); translocation heterozygotes (T1N1; T2N); individuals carrying only the $51 \mathrm{C}$ starting chromosome insertion (N1/+); or the 68E and 70A2 starting chromosome insertion (N2/+). Primers used, and expected amplification product sizes, are indicated in $\mathrm{B}$ and $\mathrm{E}$. 


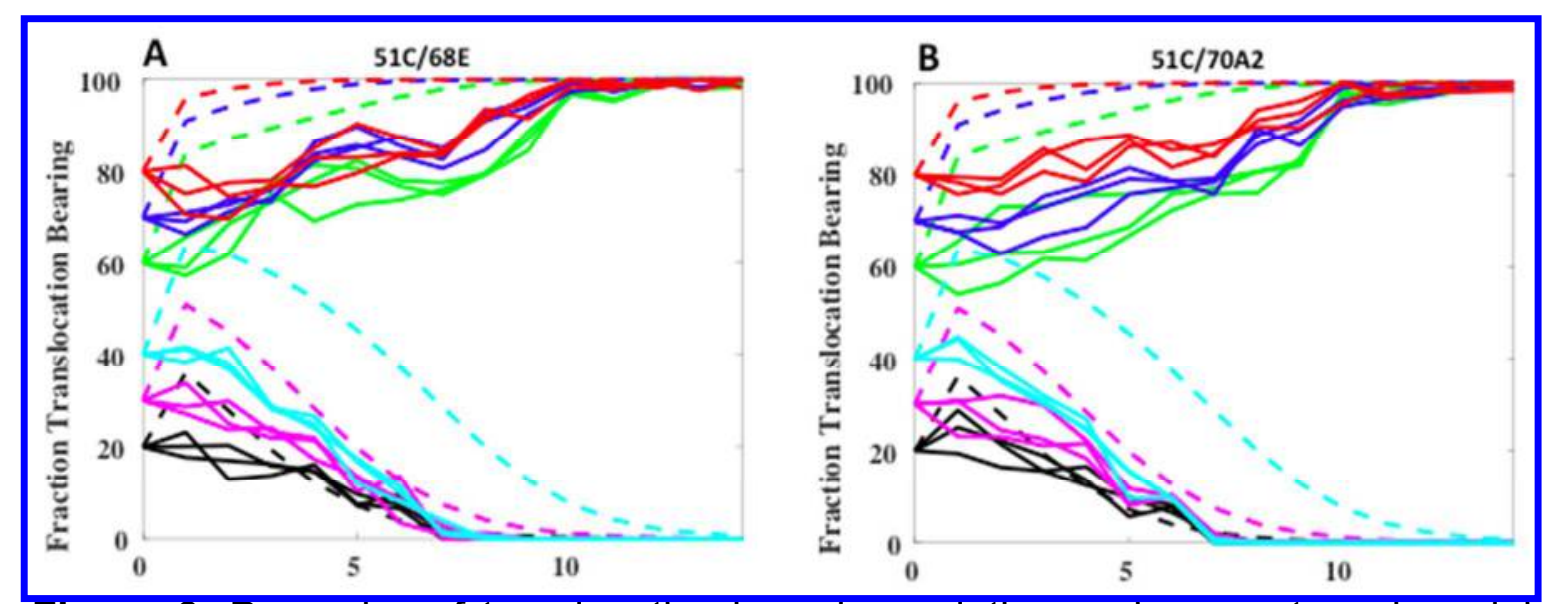

Figure 3. Dynamics of translocation-based population replacement, and model predictions in the absence of fitness costs. Population frequency of the adult population having the indicated translocation is plotted versus generation number for a number of homozygous translocation release ratios: $80 \%, 70 \%, 60 \%, 40 \%$, $30 \%$ and $20 \%$. Solid lines indicate observed population frequencies, and dashed lines indicate predicted translocation-bearing genotype frequencies for an element with no fitness cost. 


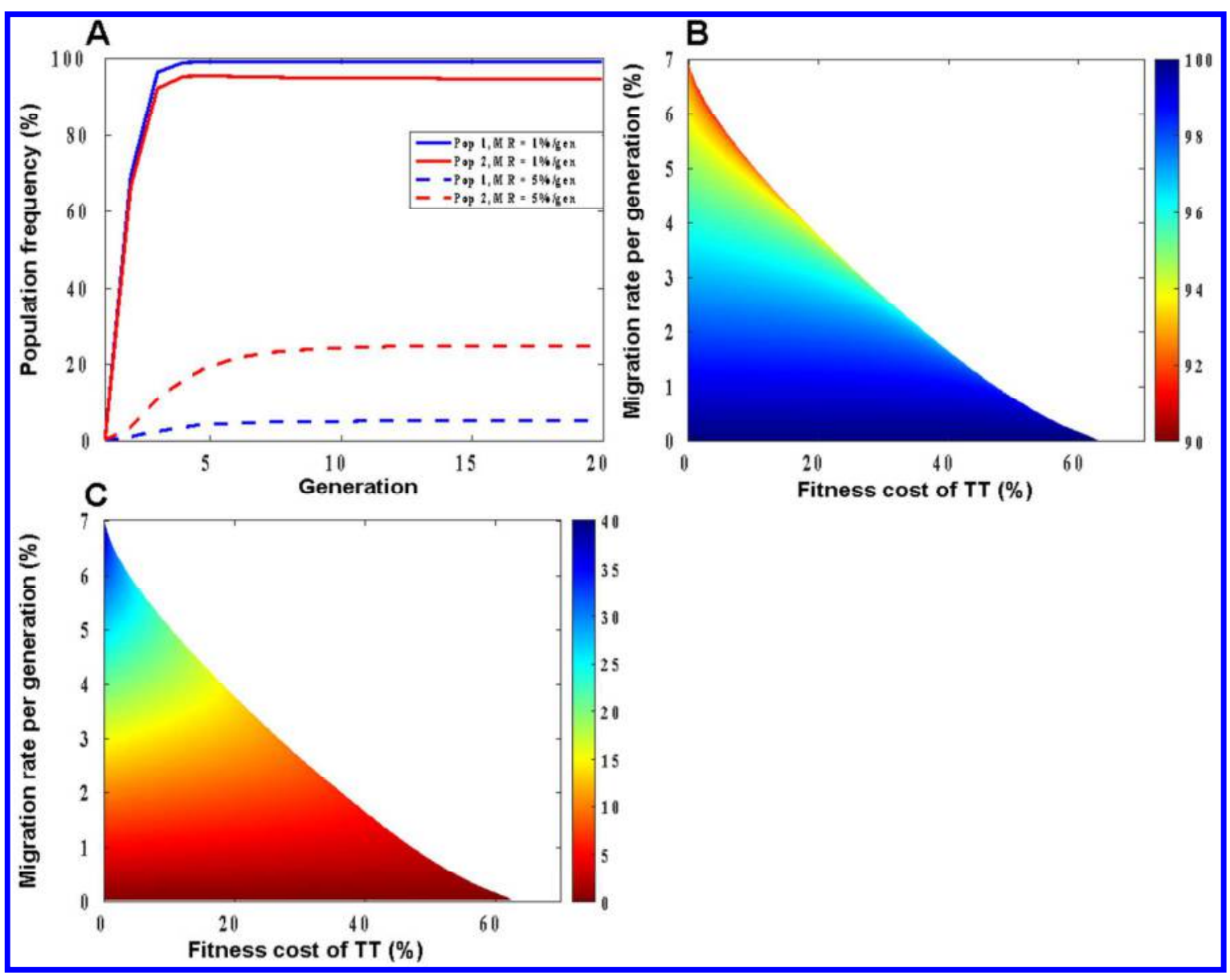

Figure 4. Translocation dynamics in a two population migration model. (A) Population frequency of a translocation with no fitness cost, introduced into population 1 using three consecutive releases of translocation-bearing homozygotes. Populations 1 and 2 are linked by a migration rate of $1 \%$ (solid lines) or $5 \%$ (dashed lines). (B,C) Equilibrium frequency of translocation bearing individuals over a range of fitness costs and migration rates for population 1 (B) or population $2(\mathrm{C})$. For both populations increasing fitness cost has little effect on the equilibrium frequency at low migration rate and increased effects at higher migration rates. In contrast, migration rate has a much stronger effect on equilibrium frequency independent of fitness cost as seen by the color gradient shifts. Note that the equilibrium frequency varies between $90-100 \%$ and $0-25 \%$ in the target population (population 1) and population 2, respectively. 


\begin{tabular}{|c|c|c|c|c|c|c|}
\hline \multicolumn{2}{|c|}{ parental genotypes } & \multirow[b]{2}{*}{ progeny genotype (\%) } & \multicolumn{2}{|c|}{ embryo survival \% } & \multicolumn{2}{|c|}{ transgene bearing adults \% } \\
\hline male & female & & predicted & observed* & predicted & observed $^{x}$ \\
\hline $\mathrm{T}_{1} / \mathrm{T}_{1} ; \mathrm{T}_{2} / \mathrm{T}_{2}$ & $\mathrm{~T}_{1} / \mathrm{T}_{1} ; \mathrm{T}_{2} / \mathrm{T}_{2}$ & $\mathrm{~T}_{1} / \mathrm{T}_{1} ; \mathrm{T}_{2} / \mathrm{T}_{2}(100 \%)$ & 100 & $\begin{array}{r}96.9 \pm 1.8(\mathrm{n}=588, \mathrm{p}=0.80) \\
96.9 \pm 0.3(\mathrm{n}=579, \mathrm{p}=0.87)\end{array}$ & 100 & $\begin{array}{l}100 \pm 0.0(\mathrm{n}=507, \mathrm{p}=1) \\
100 \pm 0.0(\mathrm{n}=592, \mathrm{p}=1)\end{array}$ \\
\hline $\mathrm{T}_{1} / \mathrm{T}_{1} ; \mathrm{T}_{2} / \mathrm{T}_{2}$ & $+/+;+/+$ & $\mathrm{T}_{1} /+; \mathrm{T}_{2} /+(100 \%)$ & 100 & $\begin{array}{c}94.6 \pm 2.2(\mathrm{n}=569, \mathrm{p}=0.62) \\
98.2 \pm 2.6(\mathrm{n}=536, \mathrm{p}=0.88)\end{array}$ & 100 & $\begin{array}{l}100 \pm 0.0(\mathrm{n}=516, \mathrm{p}=1) \\
100 \pm 0.0(\mathrm{n}=469, \mathrm{p}=1)\end{array}$ \\
\hline$+/+$ & $\mathrm{T}_{1} / \mathrm{T}_{1} ; \mathrm{T}_{2} / \mathrm{T}_{2}$ & $\mathrm{~T}_{1} /+; \mathrm{T}_{2} /+(100 \%)$ & 100 & $\begin{array}{l}90.1 \pm 1.6(\mathrm{n}=759, \mathrm{p}=0.22) \\
92.5 \pm 4.8(\mathrm{n}=410, \mathrm{p}=0.33)\end{array}$ & 100 & $\begin{array}{l}100 \pm 0.0(\mathrm{n}=507, \mathrm{p}=1) \\
100 \pm 0.0(\mathrm{n}=504, \mathrm{p}=1)\end{array}$ \\
\hline $\mathrm{T}_{1} /+; \mathrm{T}_{2} /+$ & $+/+;+/+$ & $\begin{array}{l}\mathrm{T}_{1} /+; \mathrm{T}_{2} /+(25 \%) \\
\mathrm{T}_{1} /+;+++(25 \%)^{* \pi *} \\
+/+; \mathrm{T}_{2} /+(25 \%)^{* *} \\
+/+;+1+(25 \%)\end{array}$ & 50 & $\begin{array}{l}51.2 \pm 1.6(\mathrm{n}=820, \mathrm{p}=0.90) \\
50.4 \pm 1.3(\mathrm{n}=576, \mathrm{p}=0.95)\end{array}$ & 50 & $\begin{array}{l}49.3 \pm 3.4(\mathrm{n}=825, \mathrm{p}=0.99) \\
49.5 \pm 2.4(\mathrm{n}=938, \mathrm{p}=0.99)\end{array}$ \\
\hline$t /+;+/+$ & $\mathrm{T}_{1} /+; \mathrm{T}_{2} /+$ & $\begin{array}{l}\mathrm{T}_{1} /+; \mathrm{T}_{2} /+(25 \%) \\
\mathrm{T}_{1} /+;+++(25 \%)^{* *} \\
+++; \mathrm{T}_{2}+(25 \%)^{* *} \\
+1+;+++(25 \%)\end{array}$ & 50 & $\begin{array}{l}48.3 \pm 2.8(\mathrm{n}=441, \mathrm{p}=0.77) \\
48.3 \pm 3.9(\mathrm{n}=805, \mathrm{p}=0.65)\end{array}$ & 50 & $\begin{array}{l}49.4 \pm 2.2(\mathrm{n}=877, \mathrm{p}=0.99) \\
48.5 \pm 3.4(\mathrm{n}=941, \mathrm{p}=0.99)\end{array}$ \\
\hline $\mathrm{T}_{1} /+; \mathrm{T}_{2} /+$ & $\mathrm{T}_{1} /+; \mathrm{T}_{2} /+$ & 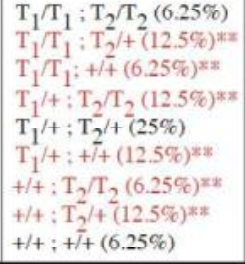 & 37.5 & $\begin{array}{l}36.2 \pm 1.8(\mathrm{n}=568, \mathrm{p}=0.84) \\
32.4 \pm 4.0(\mathrm{n}=503, \mathrm{p}=0.22)\end{array}$ & $-83 \%$ & $\begin{array}{l}80.4 \pm 6.5(\mathrm{n}=519, \mathrm{p}=0.99) \\
80.8 \pm 5.8(\mathrm{n}=463, \mathrm{p}=0.99)\end{array}$ \\
\hline
\end{tabular}

Table 1. Behavior of translocations in crosses to various genotypes. Crosses between parents of specific genotypes - wild-type $(+/+;+/+)$, translocation heterozygotes $\left(T_{1} /+; T_{2} /+\right)$, and translocation homozygotes $\left(T_{1} / T_{1} ; T_{2} / T_{2}\right)$, were carried out. Embryo survival (fifth column from right) and percentage of translocation-bearing adults (rightmost column) were independently quantified; $\chi^{2}$ statistical analysis ( $p$-values from which are shown) was carried out to determine if differences between expected and observed values were significant. The top number in each column shows results for the $51 \mathrm{C} / 68 \mathrm{E}$ translocation; the bottom number shows the results for the 51C/70A2 translocation. ${ }^{* *}$ Indicates unviable genotypes. Embryo survival was normalized with respect to percent survival ( \pm $\mathrm{SD})$ observed in the $w^{1118}$ stock used for transgenesis (methods).

Supplementary Table 1. List of primer sequences used in this study.

\begin{tabular}{|l|l|l|}
\hline $\begin{array}{l}\text { Primer } \\
\text { name }\end{array}$ & Primer sequence, 5' to 3' & Source \\
\hline P1 & $\begin{array}{l}\text { CCTAACAACTCACACCTTGCAGCGCCACCTG } \\
\text { GCCCTAGAGATCCACCAACTTTTTTGCACTG } \\
\text { C }\end{array}$ & $\begin{array}{l}\text { plZ/V5- } \\
\text { His/CAT } \\
\text { (Invitrogen) }\end{array}$ \\
\hline
\end{tabular}




\begin{tabular}{|c|c|c|}
\hline P2 & $\begin{array}{l}\text { ATTCCTAAGCATCAGTGGTTGAACCTACCTTG } \\
\text { TTGGCGTGACCAGAGACAGGTTGCGGCG }\end{array}$ & \\
\hline P3 & $\begin{array}{l}\text { AGGTTCAACCACTGATGCTTAGGAATAGGCC } \\
\text { ATGTGAAGCTGAAGGAATC } \\
\text { TATTACCCTGTTATCCCTACTAGTAGGGATAA } \\
\text { CAGGGTAATACTAGAATCCCTGGGCACAATT } \\
\mathrm{T}\end{array}$ & $\begin{array}{l}\text { pFUSEss- } \\
\text { CHIg-mG1 } \\
\text { (Invivogen) }\end{array}$ \\
\hline P5 & $\begin{array}{l}\text { CTAGTATTACCCTGTTATCCCTACTAGTAGGG } \\
\text { ATAACAGGGTAATAGTGGTTGTAAGCCTTGC } \\
\text { A } \\
\text { AAAGGATAAGAATTAGGGTTAGTCGTTTCGG } \\
\text { TGTGCCTAGTTTACCAGGAGAGTGGGAGA }\end{array}$ & $\begin{array}{l}\text { pFUSEss- } \\
\text { CHIg-mG1 } \\
\text { (Invivogen) }\end{array}$ \\
\hline $\mathrm{P} 7$ & $\begin{array}{l}\text { CGCCCACGCCATCCAACCGCCGCCGCAACC } \\
\text { TGTCTCTGGTCACGCCAACAAGGTAGGTTC }\end{array}$ & $\begin{array}{ll}\text { P3/P4 } & \text { XYZ } \\
\text { PCR }\end{array}$ \\
\hline P8 & $\begin{array}{l}\text { ATGACGTTCTTGGAGGAGCGCACCATTTTGT } \\
\text { TGCTAAAGGAAAGGATAAGAATTAGGGTT }\end{array}$ & $\begin{array}{l}\text { P5/P6 UVW } \\
\text { PCR }\end{array}$ \\
\hline $\mathrm{P} 10$ & $\begin{array}{l}\text { AAACGACTAACCCTAATTCTTATCCTTTCCTTT } \\
\text { AGCAACAAAATGGTGCGCTCCTCCAAG } \\
\text { AATGGAACTCTTCGCGGCCAGGTGGCGCTG } \\
\text { CAAGGCTCGAGGGTCGACTGATCATAATCA }\end{array}$ & $\begin{array}{l}\text { pMos-3xP3- } \\
\text { DsRed-attp } \\
\text { (addgene } \\
\text { plasmid } \\
\text { \#52904) }\end{array}$ \\
\hline $\mathrm{P} 11$ & $\begin{array}{l}\text { GGATCCGGGAATTGGGAATTGGGCAATATTT } \\
\text { AAATGGCGGCCTTGCAGCGCCACCTGGCC }\end{array}$ & $\begin{array}{l}\text { Drosophila } \\
\text { genomic DNA }\end{array}$ \\
\hline P12 & $\begin{array}{l}\text { AGCGTGTTTTTTTGGAGTGGCAAAAAAGTTGGT } \\
\text { GGATCTCTAGGGCCAGGTGGCGCTGCAA }\end{array}$ & \\
\hline P15 & $\begin{array}{l}\text { CCAACGCATTTTCCAAGCTTGTTTAAACGTGG } \\
\text { ATCTCTAGGGCCAGGTGGCGCTGCAAGG }\end{array}$ & \\
\hline P13 & $\begin{array}{l}\text { TACAAATGTGGTATGGCTGATTATGATCAGTC } \\
\text { GACCCTCGAGCCTTGCAGCGCCACCTGG }\end{array}$ & $\begin{array}{l}\text { Drosophila } \\
\text { genomic DNA }\end{array}$ \\
\hline P14 & $\begin{array}{l}\text { GAGACCGTGACCTACATCGTCGACACTAGTG } \\
\text { GATCTCTAGGGCCAGGTGGCGCTGCAAGG }\end{array}$ & \\
\hline P16 & $\begin{array}{l}\text { CCTTGCAGCGCCACCTGGCCCTAGAGATCCA } \\
\text { CGTTTAAACAAGCTTGGAAAATGCGTTGG }\end{array}$ & $\begin{array}{l}\text { Drosophila } \\
\text { genomic DNA }\end{array}$ \\
\hline P17 & $\begin{array}{l}\text { CGAAGCGCCTCTATTTATACTCCGGCGCTCG } \\
\text { TTTAAACAAAGTGGCAGGGCCCATGTGTT }\end{array}$ & \\
\hline P18 & $\begin{array}{l}\text { GAGTGGAGCACAAACACATGGGCCCTGCCA } \\
\text { CTTTGTTTAAACGAGCGCCGGAGTATAAAT }\end{array}$ & $\begin{array}{l}\text { Drosophila } \\
\text { genomic DNA }\end{array}$ \\
\hline
\end{tabular}




\begin{tabular}{|c|c|c|}
\hline P19 & $\begin{array}{l}\text { AAGCATCAGTGGTTGAACCTACCTTGTTGGC } \\
\text { GTGTCTGATGCAGATTGTTTAGCTTGTTC }\end{array}$ & \\
\hline P20 & $\begin{array}{l}\text { GCCAACAAGGTAGGTTCAACCACTGATGCTT } \\
\text { AGGAATAGGCGTGGTTGTAAGCCTTGCAT }\end{array}$ & $\begin{array}{l}\text { pFUSEss- } \\
\text { CHlg-mG1 } \\
\text { (Invivoqen) }\end{array}$ \\
\hline P21 & $\begin{array}{l}\text { CCCTGTTATCCCTACTAGTAGGGATAACAGG } \\
\text { GTAATACTAGTTTACCAGGAGAGTGGGAG }\end{array}$ & \\
\hline P22 & $\begin{array}{l}\text { TATTACCCTGTTATCCCTACTAGTAGGGATAA } \\
\text { CAGGGTAATACATGTGAAGCTGAAGGAA }\end{array}$ & $\begin{array}{l}\text { pFUSEss- } \\
\text { CHlg-mG1 } \\
\text { (Invivogen) }\end{array}$ \\
\hline P23 & $\begin{array}{l}\text { AAAGGATAAGAATTAGGGTTAGTCGTTTCGG } \\
\text { TGTGCCTAGAATCCCTGGGCACAATTTTC }\end{array}$ & \\
\hline P24 & $\begin{array}{l}\text { CAAGCGCAGCTGAACAAGCTAAACAATCTGC } \\
\text { ATCAGACACGCCAACAAGGTAGGTTCAAC }\end{array}$ & $\begin{array}{l}\text { P20/P21 UVW } \\
\text { PCR }\end{array}$ \\
\hline P25 & $\begin{array}{l}\text { ACCTACATCGTCGACACTAGTGGATCTCTAG } \\
\text { CTCGAGCTAAAGGAAAGGATAAGAATTAGGG }\end{array}$ & $\begin{array}{l}\text { P22/P23 XYZ } \\
\text { PCR }\end{array}$ \\
\hline P26 & $\begin{array}{l}\text { CCCTAATTCTTATCCTTTCCTTTAGGAATTCC } \\
\text { AACAAAATGGTGAGCAAGGGCGAGGAGC }\end{array}$ & $\begin{array}{l}\text { pAAV-GFP } \\
\text { (addgene } \\
\text { plasmid }\end{array}$ \\
\hline P27 & $\begin{array}{l}\text { TTCACTGCATTCTAGTTGTGGTTTGTCCAAAC } \\
\text { TCATCAATGTTTACTTGTACAGCTCGTC }\end{array}$ & \#32395) \\
\hline P28 & $\begin{array}{l}\text { GCCGCCGGGATCACTCTCGGCATGGACGAG } \\
\text { CTGTACAAGTAAACATTGATGAGTTTGGAC }\end{array}$ & $\begin{array}{l}\text { pMos-3xP3- } \\
\text { DsRed-attp } \\
\text { (addgene } \\
\text { plasmid } \\
\text { \#52904) }\end{array}$ \\
\hline
\end{tabular}

\section{References}

(1) Curtis, C. F. Possible Use of Translocations to Fix Desirable Genes in Insect Pest Populations. Nature 1968, 218 (5139), 368-369.

(2) Sinkins, S. P.; Gould, F. Gene Drive Systems for Insect Disease Vectors. Nat. Rev. Genet. 2006, 7 (6), 427-435.

(3) Hay, B. A.; Chen, C.-H.; Ward, C. M.; Huang, H.; Su, J. T.; Guo, M. Engineering the Genomes of Wild Insect Populations: Challenges, and Opportunities Provided by Synthetic Medea Selfish Genetic Elements. J. Insect Phvsiol. 2010, 56 (10), 14021413.

(4) Burt, A. Heritable Strategies for Controlling Insect Vectors of Disease. Philos. Trans. R. Soc. Lond. B Biol. Sci. 2014, 369 (1645), 20130432.

(5) Champer, J.; Buchman, A.; Akbari, O. S. Cheating Evolution: Engineering Gene Drives to Manipulate the Fate of Wild Populations. Nat. Rev. Genet. 2016, 17 (3), 146-159.

(6) Marshall, J. M.; Akbari, O. S. Gene Drive Strategies for Population Replacement. In Genetic Control of Malaria and Dengue; 2016; pp 169-200.

(7) Knols, B. G. J.; Bossin, H. C.; Mukabana, W. R.; Robinson, A. S. Transgenic Mosquitoes and the Fight against Malaria: Managing Technology Push in a 
Turbulent GMO World. Am. J. Trop. Med. Hyg. 2007, 77 (6 Suppl), 232-242.

(8) Marshall, J. M. The Cartagena Protocol and Genetically Modified Mosquitoes. Nat. Biotechnol. 2010, 28 (9), 896-897.

(9) Alphey, L.; Beech, C. Appropriate Regulation of GM Insects. PLoS Negl. Trop. Dis. 2012, 6 (1), e1496.

(10) Mumford, J. D. Science, Regulation, and Precedent for Genetically Modified Insects. PloS Neal. Trop. Dis. 2012, 6 (1), e1504.

(11) Reeves, R. G.; Denton, J. A.; Santucci, F.; Bryk, J.; Reed, F. A. Scientific Standards and the Regulation of Genetically Modified Insects. PLoS Neql. Trop. Dis. 2012, 6 (1), e1502.

(12) Akbari, O. S.; Bellen, H. J.; Bier, E.; Bullock, S. L.; Burt, A.; Church, G. M.; Cook, K. R.; Duchek, P.; Edwards, O. R.; Esvelt, K. M.; et al. Safeguarding Gene Drive Experiments in the Laboratory. Science 2015, 349 (6251), 927-929.

(13) Adelman, Z.; Akbari, O.; Bauer, J.; Bier, E.; Bloss, C.; Carter, S. R.; Callender, C.; Denis, A. C.-S.; Cowhey, P.; Dass, B.; et al. Rules of the Road for Insect Gene Drive Research and Testing. Nat. Biotechnol. 2017, 35 (8), 716-718.

(14) Committee on Gene Drive Research in Non-Human Organisms: Recommendations for Responsible Conduct; Board on Life Sciences; Division on Earth and Life Studies; National Academies of Sciences, Engineering, and Medicine. Gene Drives on the Horizon: Advancing Science, Navigating Uncertainty, and Aligning Research with Public Values; National Academies Press, 2016.

(15) Marshall, J. M.; Akbari, O. S. Can CRISPR-Based Gene Drive Be Confined in the Wild? A Question for Molecular and Population Biology. ACS Chem. Biol. 2018, 13 (2), 424-430.

(16) Marshall, J. M.; Hay, B. A. Inverse Medea as a Novel Gene Drive System for Local Population Replacement: A Theoretical Analysis. J. Hered. 2011, 102 (3), 336-341.

(17) Marshall, J. M.; Pittman, G. W.; Buchman, A. B.; Hay, B. A. Semele: A KillerMale, Rescue-Female System for Suppression and Replacement of Insect Disease Vector Populations. Genetics 2011, 187 (2), 535-551.

(18) Marshall, J. M.; Hay, B. A. General Principles of Single-Construct Chromosomal Gene Drive. Evolution 2012, 66 (7), 2150-2166.

(19) Gould, F.; Schliekelman, P. Population Genetics of Autocidal Control and Strain Replacement. Annu. Rev. Entomol. 2004, 49, 193-217.

(20) Davis, S.; Bax, N.; Grewe, P. Engineered Underdominance Allows Efficient and Economical Introgression of Traits into Pest Populations. J.Theor. Biol. 2001, 212 (1), 83-98.

(21) Altrock, P. M.; Traulsen, A.; Reeves, R. G.; Reed, F. A. Using Underdominance to Bi-Stably Transform Local Populations. J. Theor. Biol. 2010, 267 (1), 62-75.

(22) Altrock, P. M.; Traulsen, A.; Reed, F. A. Stability Properties of Underdominance in Finite Subdivided Populations. PLoS Comput. Biol. 2011, 7 (11), e1002260.

(23) Akbari, O. S.; Matzen, K. D.; Marshall, J. M.; Huang, H.; Ward, C. M.; Hay, B. A. A Synthetic Gene Drive System for Local, Reversible Modification and Suppression of Insect Populations. Curr. Biol. 2013, 23 (8), 671-677.

(24) Gokhale, C. S.; Reeves, R. G.; Reed, F. A. Dynamics of a Combined MedeaUnderdominant Population Transformation System. BMC Evol.Biol. 2014, 14, 98.

(25) Reeves, R. G.; Bryk, J.; Altrock, P. M.; Denton, J. A.; Reed, F. A. First Steps towards Underdominant Genetic Transformation of Insect Populations. PLoS One 2014, 9 (5), e97557.

(26) Roukos, V.; Misteli, T. The Biogenesis of Chromosome Translocations. Nat. Cell Biol. 2014, 16 (4), 293-300. 
(27) Coyne, J. A.; Allen Orr, H. Speciation; Sinauer Associates Incorporated, 2004.

(28) Warburton, D. De Novo Balanced Chromosome Rearrangements and Extra Marker Chromosomes Identified at Prenatal Diagnosis: Clinical Significance and Distribution of Breakpoints. Am. J. Hum. Genet. 1991, 49 (5), 995-1013.

(29) Ogilvie, C. M.; Braude, P.; Scriven, P. N. Successful Pregnancy Outcomes after Preimplantation Genetic Diagnosis (PGD) for Carriers of Chromosome

Translocations. Hum. Fertil. 2001, 4 (3), 168-171.

(30) Robinson, A. S. Progress in the Use of Chromosomal Translocations for the Control of Insect Pests. Biol. Rev. Camb. Philos. Soc. 1976, 51 (1), 1-24.

(31) Rickards, G. K. Orientation Behavior of Chromosome Multiples of Interchange (reciprocal Translocation) Heterozygotes. Annu. Rev. Genet. 1983, 17, 443-498.

(32) Serebrovskii, A. S. A New Possible Method of Pest Control. Zool. Zhurnal 1940, 19 (4), 618-630.

(33) Marshall, J. M.; Hay, B. A. Confinement of Gene Drive Systems to Local Populations: A Comparative Analysis. J. Theor. Biol. 2012, 294, 153-171.

(34) Barton, N. H. The Dynamics of Hybrid Zones. Heredity 1979, 43, 341.

(35) Barton, N. H.; Turelli, M. Spatial Waves of Advance with Bistable Dynamics: Cytoplasmic and Genetic Analogues of Allee Effects. Am. Nat. 2011, 178 (3), E48E75.

(36) Robinson, A. S.; Curtis, C. F. Controlled Crosses and Cage Experiments with a Translocation in Drosophila. Genetica 1973, 44 (4), 591-601.

(37) Asman, S. M.; McDonald, P. T.; Prout, T. Field Studies of Genetic Control Systems for Mosquitoes. Annu. Rev. Entomol. 1981, 26, 289-318.

(38) Curtis, C. F.; Southern, D. I.; Pell, P. E.; Craig-Cameron, T. A. Chromosome Translocations in Glossina Austeni. Genet Res. 1972, 20 (1), 101-113.

(39) Lorimer, N.; Hallinan, E.; Rai, K. S. Translocation Homozygotes in the Yellow Fever Mosquito, Aedes Aegypti. J.Hered. 1972, 63 (4), 158-166.

(40) Boussy, I. A.; Healy, M. J.; Oakeshott, J. G.; Kidwell, M. G. Molecular Analysis of the P-M Gonadal Dysgenesis Cline in Eastern Australian Drosophila Melanogaster. Genetics 1988, 119 (4), 889-902.

(41) Lorimer, N.; Lounibos, L. P.; Petersen, J. L. Field Trials with a Translocation Homozygote in Aedes Aegypti for Population Replacement. J. Econ. Entomol. 1976, 69 (3), 405-409.

(42) Foster, G. G.; Vogt, W. G.; Woodburn, T. L. Genetic Analysis of Field Trials of Sex-Linked Translocation Strains for Genetic Control of the Australian Sheep Blowfly Lucilia Cuprina (Wiedemann). Aust. J.Biol. Sci. 1985, 38 (3), 275-293.

(43) Mahon, R. J. Genetic Control of Lucilia Cuprina, Past and Prospects. In National Conference FLICS-Flystrike \& Lice IPM Control Strategies, Launceston, Tasmania; 2001; pp 225-232.

(44) Feldmann, A. M.; Sabelis, M. W. Karyotype Displacement in a Laboratory Population of the Two Spotted Spider Mite Tetranychus Urticae (Koch):

Experiments and Computer Simulations. Genetica 1981, 55 (2), 93-110.

(45) Harewood, L.; Schütz, F.; Boyle, S.; Perry, P.; Delorenzi, M.; Bickmore, W. A.; Reymond, A. The Effect of Translocation-Induced Nuclear Reorganization on Gene Expression. Genome Res. 2010, 20 (5), 554-564.

(46) Harewood, L.; Fraser, P. The Impact of Chromosomal Rearrangements on Regulation of Gene Expression. Hum. Mol. Genet. 2014, 23 (R1), R76-R82.

(47) Beumer, K. J.; Pimpinelli, S.; Golic, K. G. Induced Chromosomal Exchange Directs the Segregation of Recombinant Chromatids in Mitosis of Drosophila. Genetics 1998, 150 (1), 173-188.

(48) Yu, Y.; Bradley, A. Engineering Chromosomal Rearrangements in Mice. Nat. 
Rev. Genet. 2001, 2 (10), 780-790.

(49) Egli, D.; Hafen, E.; Schaffner, W. An Efficient Method to Generate Chromosomal Rearrangements by Targeted DNA Double-Strand Breaks in Drosophila Melanogaster. Genome Res. 2004, 14 (7), 1382-1393.

(50) Forster, A.; Pannell, R.; Drynan, L.; Cano, F.; Chan, N.; Codrington, R.; Daser, A.; Lobato, N.; Metzler, M.; Nam, C.-H.; et al. Chromosomal Translocation Engineering to Recapitulate Primary Events of Human Cancer. Cold Spring Harb. Symp. Quant. Biol. 2005, 70, 275-282.

(51) Pacher, M.; Schmidt-Puchta, W.; Puchta, H. Two Unlinked Double-Strand Breaks Can Induce Reciprocal Exchanges in Plant Genomes via Homologous Recombination and Nonhomologous End Joining. Genetics 2007, 175 (1), 21-29.

(52) Torres, R.; Martin, M. C.; Garcia, A.; Cigudosa, J. C.; Ramirez, J. C.; RodriguezPerales, S. Engineering Human Tumour-Associated Chromosomal Translocations with the RNA-Guided CRISPR-Cas9 System. Nat. Commun. 2014, 5, 3964.

(53) Chen, X.; Li, M.; Feng, X.; Guang, S. Targeted Chromosomal Translocations and Essential Gene Knockout Using CRISPR/Cas9 Technology in Caenorhabditis Elegans. Genetics 2015, 201 (4), 1295-1306.

(54) Jiang, J.; Zhang, L.; Zhou, X.; Chen, X.; Huang, G.; Li, F.; Wang, R.; Wu, N.; Yan, Y.; Tong, C.; et al. Induction of Site-Specific Chromosomal Translocations in Embryonic Stem Cells by CRISPR/Cas9. Sci. Rep. 2016, 6, 21918.

(55) Theilmann, D. A.; Stewart, S. Molecular Analysis of the Trans-Activating IE-2 Gene of Orgyia Pseudotsugata Multicapsid Nuclear Polyhedrosis Virus. Virologv 1992, 187 (1), 84-96.

(56) Gutierrez, E.; Wiggins, D.; Fielding, B.; Gould, A. P. Specialized Hepatocyte-like Cells Regulate Drosophila Lipid Metabolism. Nature 2007, 445 (7125), 275-280.

(57) Rong, Y. S.; Golic, K. G. The Homologous Chromosome Is an Effective Template for the Repair of Mitotic DNA Double-Strand Breaks in Drosophila. Genetics 2003, 165 (4), 1831-1842.

(58) Láruson, Á. J.; Reed, F. A. Stability of Underdominant Genetic Polymorphisms in Population Networks. arXiv [q-bio.PE], 2015.

(59) Sherizen, D.; Jang, J. K.; Bhagat, R.; Kato, N.; McKim, K. S. Meiotic Recombination in Drosophila Females Depends on Chromosome Continuity between Genetically Defined Boundaries. Genetics 2005, 169 (2), 767-781.

(60) Magori, K.; Legros, M.; Puente, M. E.; Focks, D. A.; Scott, T. W.; Lloyd, A. L.; Gould, F. Skeeter Buster: A Stochastic, Spatially Explicit Modeling Tool for Studying Aedes Aegypti Population Replacement and Population Suppression Strategies. PLoS Neal.Trop.Dis. 2009, 3 (9), e508.

(61) Xu, C.; Legros, M.; Gould, F.; Lloyd, A. L. Understanding Uncertainties in ModelBased Predictions of Aedes Aegypti Population Dynamics. PLoS Neal. Trop. Dis. 2010, 4 (9), e830.

(62) Lee, Y.; Marsden, C. D.; Norris, L. C.; Collier, T. C.; Main, B. J.; Fofana, A.; Cornel, A. J.; Lanzaro, G. C. Spatiotemporal Dynamics of Gene Flow and Hybrid Fitness between the M and S Forms of the Malaria Mosquito, Anopheles Gambiae. Proc. Natl. Acad. Sci. U. S. A. 2013, 110 (49), 19854-19859.

(63) Fontaine, M. C.; Pease, J. B.; Steele, A.; Waterhouse, R. M.; Neafsey, D. E.; Sharakhov, I. V.; Jiang, X.; Hall, A. B.; Catteruccia, F.; Kakani, E.; et al. Mosquito Genomics. Extensive Introgression in a Malaria Vector Species Complex Revealed by Phylogenomics. Science 2015, 347 (6217), 1258524.

(64) Dickson, L. B.; Sharakhova, M. V.; Timoshevskiy, V. A.; Fleming, K. L.; Caspary, A.; Sylla, M.; Black, W. C., 4th. Reproductive Incompatibility Involving Senegalese Aedes Aegypti (L) Is Associated with Chromosome Rearrangements. PLoS Negl. 
Trop. Dis. 2016, 10 (4), e0004626.

(65) Wise de Valdez, M. R.; Nimmo, D.; Betz, J.; Gong, H.-F.; James, A. A.; Alphey, L.; Black, W. C., 4th. Genetic Elimination of Dengue Vector Mosquitoes. Proc. Natt. Acad. Sci. U. S. A. 2011, 108 (12), 4772-4775.

(66) Deus, K. M.; Saavedra-Rodriguez, K.; Butters, M. P.; Black, W. C., 4th; Foy, B. D. The Effect of Ivermectin in Seven Strains of Aedes Aegypti (Diptera: Culicidae) Including a Genetically Diverse Laboratory Strain and Three Permethrin Resistant Strains. J. Med. Entomol. 2012, 49 (2), 356-363.

(67) Sterile Insect Technique: Principles and Practice in Area-Wide Integrated Pest Management; Dyck, V. A., Hendrichs, J., Robinson, A. S., Eds.; 2005.

(68) Harris, A. F.; McKemey, A. R.; Nimmo, D.; Curtis, Z.; Black, I.; Morgan, S. A.; Oviedo, M. N.; Lacroix, R.; Naish, N.; Morrison, N. I.; et al. Successful Suppression of a Field Mosquito Population by Sustained Release of Engineered Male Mosquitoes. Nat. Biotechnol. 2012, 30 (9), 828-830.

(69) Carvalho, D. O.; McKemey, A. R.; Garziera, L.; Lacroix, R.; Donnelly, C. A.; Alphey, L.; Malavasi, A.; Capurro, M. L. Suppression of a Field Population of Aedes Aegypti in Brazil by Sustained Release of Transgenic Male Mosquitoes. PLoS Neal. Trop. Dis. 2015, 9 (7), e0003864.

(70) Chen, C.-H.; Huang, H.; Ward, C. M.; Su, J. T.; Schaeffer, L. V.; Guo, M.; Hay, B. A. A Synthetic Maternal-Effect Selfish Genetic Element Drives Population Replacement in Drosophila. Science 2007, 316 (5824), 597-600.

(71) Akbari, O. S.; Chen, C.-H.; Marshall, J. M.; Huang, H.; Antoshechkin, I.; Hay, B. A. Novel Synthetic Medea Selfish Genetic Elements Drive Population Replacement in Drosophila; a Theoretical Exploration of Medea-Dependent Population Suppression. ACS Svnth. Biol. 2014, 3 (12), 915-928.

(72) Buchman, A.; Marshall, J.; Ostrovski, D.; Yang, T.; Akbari, O. S. Synthetically Engineered Medea Gene Drive System in the Worldwide Crop Pest, D. Suzukii. 2017.

(73) Gibson, D. G.; Young, L.; Chuang, R.-Y.; Venter, J. C.; Hutchison, C. A., 3rd; Smith, H. O. Enzymatic Assembly of DNA Molecules up to Several Hundred Kilobases. Nat. Methods 2009, 6 (5), 343-345.

(74) Bischof, J.; Maeda, R. K.; Hediger, M.; Karch, F.; Basler, K. An Optimized Transgenesis System for Drosophila Using Germ-Line-Specific phiC31 Integrases. Proc. Natl.Acad Sci. U.S.A. 2007, 104 (9), 3312-3317.

(75) Kyrchanova, O.; Toshchakov, S.; Podstreshnaya, Y.; Parshikov, A.; Georgiev, P. Functional Interaction between the Fab-7 and Fab-8 Boundaries and the Upstream Promoter Region in the Drosophila Abd-B Gene. Mol. Cell. Biol. 2008, 28 (12), 4188-4195.

(76) Curtis, C. F.; Robinson, A. S. Computer Simulation of the Use of Double Translocations for Pest Control. Genetics 1971, 69 (1), 97-113. 


\section{For Table of Contents Use Only}

Engineered reciprocal chromosome translocations drive high threshold, reversible population replacement in Drosophila

Anna B. Buchman*\#, Tob Ivy*, John M. Marshall ${ }^{\$}$, Omar \$. Akbari*\#\&, and Bruce A. Hay *\&

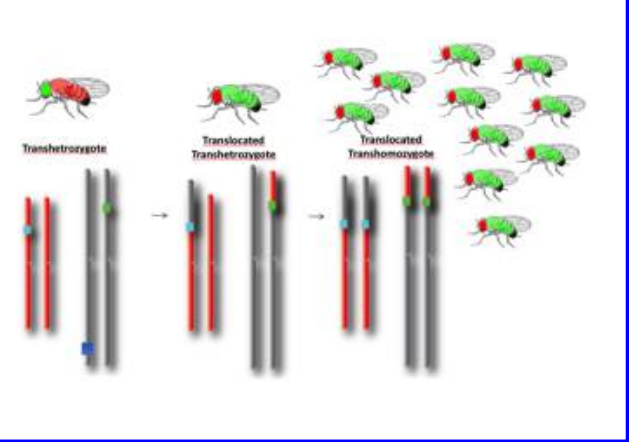

